\section{AB1062 RISK FACTORS FOR MORTALITY AFTER TUBERCULOUS ARTHRITIS}

Y.-C. Chen. Rheumatology, Kaohsiung Chang Gung Memorial Hospital, Kaohsiung, Taiwan, Province of China

Background: Tuberculosis arthritis is an emerging international problem despite advances in the methods of diagnosis and treatment; it is still prevalent in developing countries and on the increase in developed ones.

Objectives: Patients with tuberculous (TB) arthritis increase mortality This study investigated to search for the risk factors of mortality in TB arthritis.

Methods: We retrospectively reviewed patients with TB arthritis who admit to Kaohsiung Chang Gang Memorial Hospital Jauary 2001 to December 2015. The long-term outcomes of the patients were analysed.

Results: A total of 281 patients (124 females and 17 males; mean age: 64.86 \pm 14.51 years) were enrolled. At the end of the study, 229 patients were alive and 52 had died. The mean follow up period were $8.31 \pm 4.52$ years. The patients who were old age $(p=0.004, H R=1.038,95 \% C l 1.012-1.065)$, liver disease $(p=0.036$, $\mathrm{HR}=2.571,95 \% \mathrm{Cl} 1.062-6.224)$ and underlying cancer $(\mathrm{p}=0.001, \mathrm{HR}=3.640$, $95 \% \mathrm{Cl} 1.725-7.679$ ) had a significantly higher mortality rate than others.

Abstract AB1062 - Table 1

\begin{tabular}{lcccccc}
\hline & $\begin{array}{c}\text { Regression } \\
\text { coefficient }\end{array}$ & SE & $\begin{array}{c}\mathrm{P} \\
\text { value }\end{array}$ & HR & \multicolumn{2}{c}{$95.0 \%$ Cl for HR } \\
\hline Age & 0.037 & 0.013 & 0.004 & 1.038 & 1.012 & 1.065 \\
Gender & -0.057 & 0.320 & 0.859 & 0.945 & 0.505 & 1.769 \\
BMl & -0.025 & 0.044 & 0.573 & 0.976 & 0.896 & 1.063 \\
cancer & 1.292 & 0.381 & 0.001 & 3.640 & 1.725 & 7.679 \\
Steroid & -0.173 & 0.398 & 0.664 & 0.841 & 0.385 & 1.836
\end{tabular}

Conclusions: When we treat TB arthritis, old age and underlying liver and cancer history should paid more attention to decrease the risk of mortality.

\section{REFERENCE:}

[1] LongQ, LiY, WangYetal(2008)BarrierstoaccessingTBdiagnosis forrural-tourbanmigrantswithchroniccoughinChongqing, China: a mixed methods study. BMC Health Serv Res 8:202

Acknowledgements: We thank Kaohsiung CGMH for data support

Disclosure of Interest: None declared

DOI: 10.1136/annrheumdis-2018-eular.5428

\section{Fibromyalgia}

\section{AB1063 SWALLOWING DIFFICULTY IN FIBROMYALGIA: REAL OR MYTH?}

E. Umay, E. Gurcay, O. Karaahmet, A. Hasturk, D. Dulgeroglu Erdogdu. University of Health Sciences Ankara Yildirim Beyazit Education and Research Hospital, Ankara, Turkey

Background: Fibromyalgia Syndrome (FMS) is a chronic disease that the most common disease in medical practice. This syndrome is including some general symptoms such as chronic pain, fatique, tenderness and sleep disturbance, that can be seen in many disorders. Other than these, there are symptoms such as dysphagia, which are reported less frequently but have serious consequences such as aspiration. Studies in literature have been reported that this may not be a real swallowing difficulty and may be a symptom of somatization or may be due to adverse events such as drug side effects.

Objectives: This study was aimed to reveal the presence of dysphagia with objective diagnostic tests in FMS patients.

Methods: This study was conducted on 165 subjects. Patients ( $n=83$ ) with FMS regardless of whether the complaint is difficulty swallowing as well as had ages between $18-65$ were included in the study. Healthy volunteers $(n=82)$ consisting of hospital staff and relatives of patients and sex, age and body mass indexmatched with patients were included as control group.

Demographic and disease characteristics were recorded. Fibromyalgia Impact Questionnaire (FIQ)was used to assess severity of disease, functional disability and specific quality of life of patients. Also, the general quality of life of patients was evaluated with Short Form-36 (SF-36). All subject were evaluated with clinical screen test (10-item eating assessment tool=Eat-10) flexible fiberoptic endoscopic evaluation of swallowing (FEES) and electrophysiologic evaluation(EE) as well as ultrasonography (US).
The patients were divided into two groups according to the presence of complaints of difficulty in swallowing, which was taken with eat-10 as group 1 (with dysphagia) and group 2 (with normal swallowing). Patients ve healthy volunteers (group 3) were compared in terms of swallowing evaluation methods.

Results: Twenty-one $(25.3 \%)$ of the 83 patients were defined with dysphagia (group 1) as well as $62(74.7 \%)$ patients with normal swallowing (group 2) according to the eat- 10 scale. The groups were similar in terms of demographic characteristics $(p>0.05)$.

The mean disease severity of the all patients was between moderate and severe levels as well as there was a mild impact on quality of life. Disease severity and quality of life by using FIQ and SF-36 were significantly worse in patients with dysphagia (group 1) compared with normal swallowing patients (group 2) $(p<0.05)$.

None of the subjects had dysphagia with endoscopic evaluation. All subjects could drink $20 \mathrm{ml}$ of water which normal dysphagia limit.

Swallowing intervals in patients groups were significantly prolonged compared to healthy volunteers $(p<0.05)$. There was no significant difference between the patient groups in swallowing intervals $(p>0.05)$.

Ultrasonographic measurement results in healthy subjects were higher than patient groups $(p<0.05)$. But ultrasonographic values of patients groups were simi$\operatorname{lar}(\mathrm{p}>0.05)$.

Conclusions: Swallowing functions and structures are affected in patients with FMS, even though there are no symptoms of swallowing difficulty. Hence, while the main symptoms such as pain, fatigue and sleep disturbance are being questioned, a detailed evaluation including swallowing should be performed.

Disclosure of Interest: None declared

DOI: 10.1136/annrheumdis-2018-eular.2451

\section{AB1064 EXPLORING CEREBROSPINAL FLUID PROTEOME IN FIBROMYALGIA}

E. Ossipova ${ }^{1}$, P. Emami Khoonsari ${ }^{2}$, J. Lengqvist ${ }^{1}$, E. Kosek ${ }^{3}$, D. Kadetoff ${ }^{3}$, P. J. Jakobsson', K. Kultima ${ }^{2}$, J. Lampa'. ' Medicine, Karolinska Institutet, Stockholm; ${ }^{2}$ Medical Sciences, Uppsala University, Uppsala; ${ }^{3}$ Clinical Neuroscience, Karolinska Institutet, Stockholm, Sweden

Background: Fibromyalgia (FM) is a heterogeneous disease of unknown etiology characterised by chronic widespread pain that affects up to $4 \%$ of population Overlapping and heterogeneous symptoms of various chronic pain conditions complicate their diagnosis, emphasising the need for more specific biomarkers to improve the diagnosis and understand the disease mechanisms. Cerebrospinal fluid (CSF) flows in the ventricles within the brain and diffuses over the brain and spinal cord. Due to direct contact of CSF with CNS, content of CSF reflects biochemical changes in CNS making it an excellent source for biomarker discovery. Objectives: In current study we aim to explore CSF proteome of FM patients utilising quantitative proteomics method based on stable isotope labelling of CSF peptides combined with multivariate data analysis (MVDA) in order to monitor the dynamics of the proteome while comparing to the CSF proteomes in patients with rheumatoid arthritis (RA) and other neurological diseases (OND) and define the potential biomarker candidates in $\mathrm{FM}$. We also investigate, which protein products have been found in human CSF with respect to known "pain" genes, ${ }^{1}$ human CSF proteome explored if these proteins represent any clear subgrouping of "pain proteins".

Methods: CSF samples from patients with FM, RA and control OND group were collected by lumbar puncture and equal aliquots were subsequently reduced, alkylated and digested by trypsin. Obtained peptides were labelled by stable isotopes and mixed prior sample fractionation. The degree of sample complexity was reduced by off-line peptide separation using HPLC instrumentation. Obtained 80 peptide fractions were combined into 10 fractions across the gradient area. Fractions were analysed by LC-MS/MS, proteins in acquired data were identified and quantified, and data was analysed using MVDA.

Results: Out of the 1422 proteins identified, 855 proteins were included in the quantitative data analysis. Comparing FM, RA and OND groups to each other using univariate testing we found 53 statistical significant proteins (q-value $<0.05$ ). Six out of these have been reported as "pain proteins" (Complement C4-A, Prostaglandin-H2 d-isomerase, Apolipoprotein D, Granulins, Pro-cathepsin H, and BMP and activin membrane-bound inhibitor homolog).

Conclusions: We have employed quantitative proteomics methods combined with extensive bioinformatics data analysis to investigate differences in proteome profiles in CSFs obtained from patients with FM, and identified six differentially expressed pain proteins of various functions in CSF of FM patients. The involvement of these proteins in the disease pathogenesis as well use of the identified proteins as potential biomarkers should be investigated.

\section{REFERENCE:}

[1] Ultsch A, Kringel D, Kalso E, Mogil JS, Lötsch J. A data science approach to candidate gene selection of pain regarded as a process of learning and neural plasticity. Pain. 2016. 
Acknowledgements: The research leading to these results has received funding from the European Union Seventh Framework Programme (FP7/2007 - 2013) under grant agreement no 602919 (GLORIA), Swedish Medical Research Council, Åke Wiberg Foundation and Magnus Bergvalls Foundation and Uppsala Berzelii Technology Centre for Neurodiagnostics, with financing from the Swedish Governmental Agency for Innovation Systems.

Disclosure of Interest: None declared

DOI: 10.1136/annrheumdis-2018-eular.5253

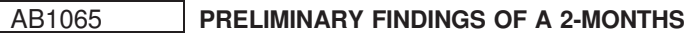 ACUPUNCTURE INTERVENTION ON SYMPTOMATOLOGY AND QUALITY OF LIFE IN PATIENTS WITH FIBROMYALGIA}

M. Di Carlo, G. Beci, F. Salaffi. Rheumatological Clinic, Università Politecnica delle Marche, Jesi, Italy

Background: Acupuncture is frequently used in the treatment of different chronic pain conditions. In Fibromyalgia (FM) the evidences available are someway conflicting, and the correct positioning of such kind of therapy has not yet precisely defined.

Objectives: To assess the response to a single course of acupuncture in patients with FM non-responsive to the usual pharmacological treatment.

Methods: Consecutive FM patients with unsatisfactory response or intolerance to the pharmacologic treatment (duloxetine and/or pregabalin) were involved in this study. Unsatisfactory response was defined by the presence of a revised Fibromyalgia Impact Questionnaire (FIQ-R) total score $>40$ and of a Patient Health Questionnaire 15 (PHQ15) $\geq 5$ after 3 months of stable pharmacological treatment. Acupuncture treatment consisted in 8 weekly sessions. The acupuncture formula, according to the Traditional Chinese Medicine indications, included the following points: LV3 +LI4 (to move Qi), ST36 +CV6+CV12 (to tonify Qi and Blood), GV20 (to raise Qi), and Ex-HN-3 (Yintang) (to calm the Shen), with acupuncture needle $0.25 \times 25 \mathrm{~mm}$ with guide tube (Huanqiu). For each session needles were retained for $30 \mathrm{~min}$. At baseline (before the first session) and at the end of the treatment course (after the eighth session) were collected the number of tender points (TP) and patient-reported outcomes (PROs). Differences between baseline and end of the acupuncture treatment were evaluated through the Wilcoxon test, results expressed in median values with $95 \%$ confidence interval $(\mathrm{Cl})$. Results: Thirty-four subjects were enrolled in the study. Thirty-two patients (29 women, 3 men, mean age 49 years, range 18-72 years) completed the acupuncture treatment. In two patients (one woman and one man) the acupuncture therapy was stopped at the second session for poor tolerance to the needles. Eleven patients were in pharmacological therapy with pregabalin, nine with duloxetine, while 12 resulted intolerant both to pregabalin and duloxetine. From baseline, after the 2 months of acupuncture treatment, different parameters showed a significant improvement. Particularly, it has been observed a significant reduction in the TP number $(17$ [95\% Cl 16-18] à $10[95 \% \mathrm{Cl} 8-12]$; $\mathrm{p}<0.0001)$, in the somatic symptoms assessed with the PHQ15 (13.5 [10.0-17.0] à 7.0 [6.0-10.0]; $p<0.0001)$, but also in the FIQ-R total score (61.5 [39.8-70.3] à [30.2-66.5]; $p=0.0029)$, in the Fibromyalgia Activity Score (FAS) (6.7 [4.8-7.7] à 4.6 [Cl 3.2-6.1]; $p=0.0017)$, and in the Self-Assessment Pain Scale (SAPS) (4.5 [3.8-5.6] à 3.2 [2.9-4.2]; $\mathrm{p}=0.0192$ ). Interestingly, acupuncture revealed a good effect even in the neuropatic-like features of pain, measured by the painDETECT questionnaire (19.0 [15.0-25.0] à 14.5 [10.9-17.0]).

Conclusions: A 2 months acupuncture treatment provides an important global improvement in the health status in FM patients refractory/intolerant to the pharmacologic therapy. The strongest ameliorations are represented by the reduction in the TP number and in the somatic symptoms.

\section{REFERENCES:}

[1] Häuser W, Clauw DJ, Fitzcharles MA. Treat-to-Target Strategy for Fibromyalgia: Opening the Dialogue. Arthritis Care Res 2017; doi: 10.1002/ acr.22970.

[2] Langhorst J, Heldmann P, Henningsen P, et al. Complementary and alternative procedures for fibromyalgia syndrome: Updated guidelines 2017 and overview of systematic review articles. Schmerz 2017; doi: 10.1007/ s00482-017-0206-1.

Disclosure of Interest: None declared DOI: 10.1136/annrheumdis-2018-eular.7016

\section{AB1066 DIABETES MELLITUS TYPE 2 RISK ASSESSMENT IN FIBROMYALGIA WOMEN}

O.V. Dzekan ${ }^{1}$, M. Stanislavchuk ${ }^{2} .{ }^{1}$ Rheumatology, Vinnytsya Regional Clinical Hospital; ${ }^{2}$ Internal Medicine, National Pirogov Memorial Medical University, Vinnytsya, Ukraine

Background: Fibromyalgia (FM) female are less physically active than sedentary healthy women. ${ }^{1}$ Low physical activity (PA) is among the leading causes of the major noncommunicable diseases, including diabetes mellitus type 2 (DM2). ${ }^{2-3}$ An increased prevalence rate of FM in DM2 patients was revealed. ${ }^{4-5}$ Risk of the development of DM2 in FM is unknown.

Objectives: The purpose of this study was to assess the risk of DM2 development in FM women.

Methods: The study involved 67 FM women ${ }^{A C R} 1990$ and 2010 criteria) aged 42.6 \pm 7.8 (M $\pm S D$ ) yrs without known diabetes, and 51 healthy controls (HCs) (all women) aged $44.8 \pm 7.3$ years. All participants were asked to complete a modified version of the Finnish Diabetes Risk Score (FINDRISC), which evaluates age, body mass index, waist circumference, current antihypertensive medication, frequency of fruit and vegetable consumption, physical activity, personal history of high blood glucose, and family history of DM2. ${ }^{6}$ Fasting plasma glucose (FPG), oral glucose tolerance test (OGTT) and/or glycated haemoglobin ( $\mathrm{HbA1C}$ ) values were collected from all subjects to determine their glucometabolic state. Prediabetes was diagnosed by the presence of impaired FPG ( $\geq 100 \mathrm{mg} / \mathrm{dL}$ to $<126 \mathrm{mg}$ / $\mathrm{dL}$ ), impaired OGTT ( $\geq 140 \mathrm{mg} / \mathrm{dL}$ to $<200 \mathrm{mg} / \mathrm{dL}$ ) and/or impaired $\mathrm{HbA} 1 \mathrm{c}$ $(\geq 5.7 \% \text { to } \leq 6.4 \%)^{7}$

Results: According to the FINDRISC questionnaire low risk of DM2 in the next 10 years was found in $14.92 \%$ of FM women, intermediate risk -in $22.39 \%$, moderate risk - in $29.85 \%$, high risk - in $23.88 \%$, a very high risk - in $8.96 \%$ of patients In the group of HCs low risk of DM2 was found in $19.61 \%$ of women, intermediate risk -in $39.22 \%$, moderate risk - in $21.57 \%$, high risk -in $15.68 \%$, a very high risk - in $3.92 \%$ of subjects. Therefore, in most of FM female $(53,73 \%)$ risk of DM2 was detected as moderate-to-high, while in the majority of HCs $(60.79 \%)$ it was detected as intermediate-to- moderate. Prediabetes was diagnosed in $11.94 \%$ of FM female compared to $5.88 \%$ among healthy women.

Conclusions: FM women are found to have increased risk of DM2 development compared to healthy women.

\section{REFERENCES:}

[1] McLoughlin MJ, Colbert LH, Stegner AJ, et al. Are women with fibromyalgia less physically active than healthy women? Med Sci Sports Exerc 2011;43:905-12.

[2] Global action plan for the prevention and control of noncommunicable diseases 2013-2020. Geneva: World Health Organization; 2013 (http://www. who.int/nmh/publications/ncd-action-plan/en/, accessed 12 June 2015).

[3] Brugnara L., Murillo S., Novials A., et al. Low Physical Activity and Its Association with Diabetes and Other Cardiovascular Risk Factors: A Nationwide, Population-Based Study. PLoS One. 2016; 11(8): e0160959. Published online 2016 Aug 17. doi: 10.1371/journal.pone.0160959

[4] M. Tishler, M. Vazina-Amit, Y. Ramot, T. Smorodin, M. Koffler, and B. Fishel. Fibromyalgia in diabetes mellitus. Rheumatology International, vol. 23, no. 4, pp. 171-173, 2003.

[5] M. N. Yanmaz, M. Mert, and M. Korkmaz. The prevalenceof fibromyalgia syndrome in a group of patients with diabetes mellitus. Rheumatology International. April 2012, Volume 32, Issue 4, pp 871-874.

[6] Lindström J, Tuomilehto J. The diabetes risk score: a practical tool to pre dict type 2 diabetes risk. Diabetes Care. 2003;26:725-731.

[7] American Diabetes Association. Standards of medical care in diabetes2014. Diabetes Care. 2014;37 Suppl 1:S14-S80.

Disclosure of Interest: None declared

DOI: 10.1136/annrheumdis-2018-eular.7425

\section{AB1067 PREVALENCE OF FIBROMYALGIA IN PATIENTS WITH PAINFUL KNEE OA}

O.V. Dzekan ${ }^{1}$, M. Stanislavchuk ${ }^{2} .{ }^{1}$ Rheumatology, Vinnytsya Regional Clinical Hospital; ${ }^{2}$ Rheumatology, National Pirogov Memorial Medical University, Vinnytsya, Ukraine

Background: Fibromyalgia (FM) has been conceptualised as a central nervous system (CNS) disorder, characterised by augmented sensory processing and inability to modulate pain effectively[. ${ }^{1}$ Global mean prevalence of $\mathrm{FM}$ is $2.7 \%$ with female/male ratio of $4: 1$. Several studies highlight the importance of central sensitisation (CS) as a significant manifestation of neuropathic pain symptoms in knee osteoarthritis (OA) ${ }^{2-3}$ Possible similar mechanisms of chronic pain maintenance in $\mathrm{OA}$ - structural pathology, and FM - central sensitivity syndrome, provoke 